\title{
The Pathogenesis and Prevention of Diabetic Neuropathy and Nephropathy
}

\author{
Douglas Greene
}

The chronic complications of diabetes are thought to be caused by an interaction between hyperglycemia, or other metabolic consequences of insulin deficiency, and independent genetic or environmental factors that are poorly defined. Several potentially relevant biochemical sequelae to hyperglycemia have been identified in tissue susceptible to diabetic complications. Among these, a rise in tissue sorbitol secondary to concentration-dependent activation of polyol pathway activity by glucose, and an accompanying fall in tissue myo-inositol and Na-K-ATPase activity have recently been linked to a self-reinforcing cyclic metabolic defect that accounts for rapidly reversible slowing of conduction in peripheral nerve in diabetes. Impaired Na-K-ATPase activity also appears to be responsible for intracellular $\mathrm{Na}^{+}$accumulation and resultant localized axonal paranodal swelling that characterizes diabetic neuropathy in both humans and laboratory animals. These swellings are thought to be responsible for the subsequent disruption of the nodal apparatus (axo-glial disjunction) and some component of the loss of large and small myelinated fibers. Recent studies have suggested that microvascular insufficiency may also contribute to diabetic neuropathy, especially in non-insulin-dependent diabetes. Aldose reductase activity is concentrated in endoneurial vessels, and similar biochemical mechanisms (ie, sorbitol accumulation, myo-inositol deficiency, and impaired Na-K-ATPase activity) are thought to be operative in the endoneurial microvessels in diabetes. Administration of an aldose reductase inhibitor to patients with diabetic neuropathy is associated with repair of damaged nerve fibers and the appearance of newly generated fibers, presumably secondary to metabolic correction within the nerve fibers themselves or their supporting microvasculature. Components of this same biochemical pathway have now been identified in the renal glomerulus and the retina, which may implicate this metabolic sequence in the pathogenesis of multiple diabetic complications.

( 1988 by Grune \& Stratton, inc.

$\mathbf{O}$ NE OF THE MOST persistent, controversial, and important questions concerning diabetes is whether the so-called glucose hypothesis of diabetic complications is correct. Essentially, the hypothesis is that hyperglycemia and/or insulin deficiency leads to secondary biochemical abnormalities in target tissues, which are followed by alterations in the functions of these tissues, the appearance of structural lesions, and, ultimately, clinical complications. In addition, at each point in this sequence of events, independent genetic or environmental variables seem to alter the expression of these complications. The results of one study indicate that male diabetics are more likely to develop diabetic neuropathy than their female counterparts, despite similar duration of disease and degree of hyperglycemia, and that taller individuals are more likely to develop diabetic neuropathy than shorter ones, even after correction of glycosylated hemoglobin and duration of diabetes. ${ }^{2}$ Other studies indicate that the development of diabetic neuropathy seems to be accelerated by alcohol consumption and nutritional deficiencies. ${ }^{2,3}$

If the modified glucose hypothesis of diabetic complications is correct, there should first be a logical sequence of events that starts with glucose and ends with a clinical complication. Secondly, this hypothesis should in some way address the well-known clinical variability of diabetic complications. Diabetic neuropathy embraces a tremendously varied spectrum of disease. A patient with an acute onset of ptosis and diplopia who develops an isolated third nerve palsy presents an example of diabetic cranial neuropathy, a condition that has a good chance of resolving within a year or so. ${ }^{4}$ In contrast to this, the more common chronic indolent distal symmetric polyneuropathy arising over 10 to 15 years tends to be progressive, despite temporary periods of remission. ${ }^{5}$ Symptoms such as pain or paresthesias may resolve, but sensory deficits generally worsen from paresthesia to anesthesia.

\section{AUTONOMIC NEUROPATHY}

Autonomic neuropathy, which can involve virtually any of the visceral organs, tends to be progressive. One patient may present with severe gastroparesis with little clinical evidence of cardiovascular autonomic neuropathy, and another may present with orthostatic hypotension without obvious delayed gastric emptying.

Widespread subclinical autonomic dysfunction is present in almost all patients with symptomatic autonomic neuropathy. Again, the disease may express itself differently in each person. The chronic progressive forms of diabetic neuropathy, distal symmetric polyneuropathy and autonomic neuropathy, exhibit widespread loss of large and small myelinated and unmyelinated fibers, as does autonomic neuropathy. Indeed, it is not uncommon for patients to lose two thirds or more of their myelinated nerve fibers. This loss is accompanied by proliferation of endoneurial connective tissue and a series of vascular abnormalities, including thickening of the vascular basement membrane, capillary closure, and thrombotic occlusion within the peripheral nervous system. ${ }^{6}$

Is this chronic destructive process related to blood glucose abnormalities? A study by Troni et al demonstrated that reducing hyperglycemia acutcly improves nerve conduction velocity (Fig 1). ${ }^{7}$ Other studies have had similar results, suggesting that glucose or a glucose metabolite may acutely impair nerve function in diabetics. ${ }^{8}$ But what is the biochemical mechanism responsible for changes in nerve function?

From the University of Michigan Medical Center, Ann Arbor, Michigan.

Address reprint requests to Douglas Greene, MD. University of Michigan Medical Center, 5510 Medical Science Research Building \#1, Ann Arbor, MI 458109-0800.

(c) 1988 by Grune \& Stratton, Inc.

0026-0495/88/3702-1005\$03.00/0 

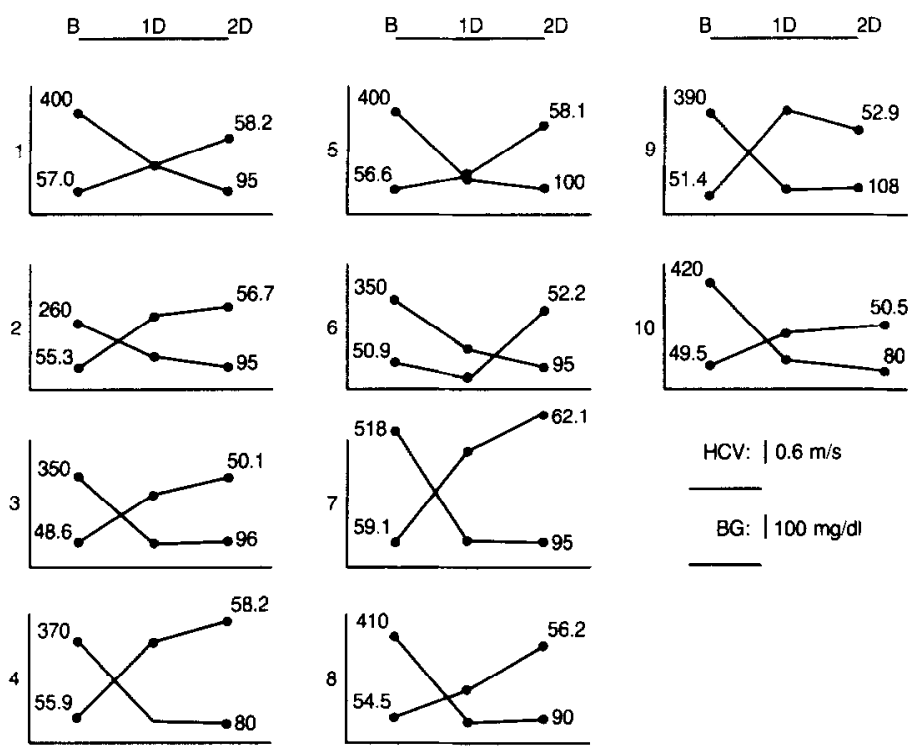

HCV: $\mid 0.6 \mathrm{~m} / \mathrm{s}$

BG: $\mid 100 \mathrm{mg} / \mathrm{dl}$

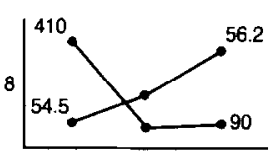

Quantitatively, the most impressive abnormality in nerve metabolism in diabetes is a diversion of glucose metabolism from oxidative to reductive pathways. Under normal circumstances, glucose is primarily oxidized to carbon dioxide and water for efficient derivation of energy in the form of adenosine triphosphate (ATP) (Fig 2, left panel). When hyperglycemid is present, excess glucose enters the nerve because its transport is neither rate-limiting for metabolism nor insulin dependent. Glycolytic pathways become saturated and there is a marked increase in the reduction of sorbitol by the enzyme aldose reductase once glucose levels are elevated within peripheral nerves. The sorbitol is then metabolized to fructose by sorbitol dehydrogenase.

\section{THE SORBITOL PATHWAYS}

Is there a link between abnormal glucose metabolism through the sorbitol pathways and reversible abnormalities in nodal (nodes of Ranvier) function? It has been established that the enzyme aldose reductase is localized in the nodes of Ranvier, ${ }^{9}$ but to understand the effects of sorbitol pathway on nerve conduction, it is first necessary to understand another biochemical abnormality of diabetic peripheral
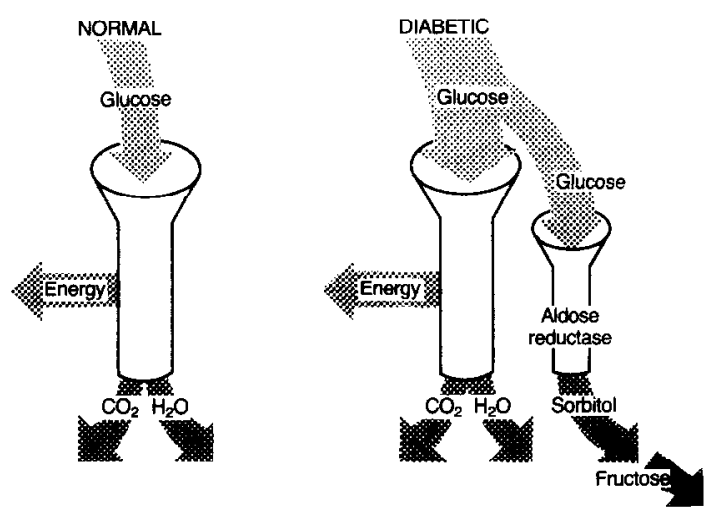

Fig 2. Schematic depiction of glucose metabolism in normal and diabetic nerve.
Fig 1. Changes in mean conduction velecity over the H-reflex pathway ( $n$-HVC) and blood clueoes during artificiel endocrine pencreas treatment in indtvidual patients. B, baseline; D, determination; BG, blood glucose. Reprinted with permission.? nerve. When the sorbitol pathway was first intensively studied in diabetic peripheral nerve, independent studies were being conducted on the metabolism of myo-inositol in peripheral nerve. In studies of diabetic rats, myo-inositol levels were decreased in peripheral nerve, but administration of insulin prevented this decrease and improved nerve conduction velocity. ${ }^{10}$ Furthermore, myo-inositol depletion but did not lower elevated nerve glucose and sorbitol levels, restored normal nerve conduction velocity. Understandable, the so-called sorbitol hypothesis and the myo-inositol hypothesis were originally considered to be two independent hypotheses that attempted to explain acute and reversible changes in nerve conduction in experimental diabetic neuropathy. However, increasingly compelling evidence suggested that the biochemical defects were tightly intertwined. ${ }^{11}$

In virtually every tissue prone to diabetic complications, a rise in sorbitol level is accompanied by evidence of myoinositol depletion within the tissue; however, the exact mechanism for this seesaw interrelationship is still not clear. The observation that myo-inositol depletion occurs in diabetic complications preceded our recognition that myo-inositol metabolism plays a unique role in cellular regulation. Myoinositol belongs to a class of compounds called phosphoinositides, which are metabolically labile phospholipids consisting of myo-inositol in phosphodiesterase linkage to a glycerol moiety containing two fatty acids, which are usually arachidonic and stcaric acid.

\section{PHOSPHOINOSITIDE METABOLISM}

Phosphoinositide hydrolysis is initiated by a variety of specific receptor mediated stimuli, yielding two important metabolically active intracellular metabolites, diacylglycerol and inositol polyphosphates, which modulate an array of ccll functions. Diacylglycerol, a lipid-soluble metabolite, is an activator of protein kinase $\mathrm{C}$, which phosphorylates a large number of intracellular proteins, thus modulating their activity. Inositol polyphosphates, the water-soluble derivatives of phosphoinositides, have marked effects on intracellular calcium metabolism. Therefore, myo-inositol through its incor- 
Fig 3. Schematic representation of a node of Ranvier, illustrating the sodium pump (loft) and voltagedopendent sodium channels (right) embedded in the axon membrane.

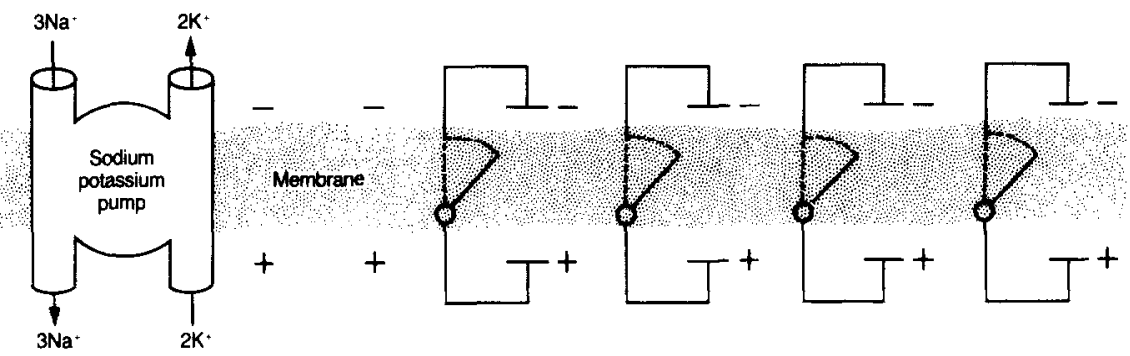

poration into phospholipids plays a very important role in transmembrane signaling and in the regulation of intracellular metabolism. Thus, hyperglycemia stimulates polyol pathway activity, which leads to myo-inositol depletion, which in turn leads to derangements in phosphoinositide-mediated transmembrane signal transduction, which then leads to interference with cell function.

Do alterations in phosphoinositide metabolism contribute to abnormal function of the nodes of Ranvier? A node of Ranvier contains three important functional elements: (1) the axonal membranc, (2) a sodium pump or Na-K-ATPase that generates an electrochemical sodium gradient across the membrane, and (3) a series of voltage-sensitive sodium channels that become permeable to sodium in the presence of a depolarizing voltage and discharge the gradient generated by the Na-K-ATPase (Fig 3), As has been demonstrated in a series of biophysic and neurochemical studies, Na-KATPase activity is reduced in diabetic nerve, but if an accumulation of sorbitol is prevented by means of an aldose reductase inhibitor, the impairment of Na-K-ATPase is prevented. In addition, if sorbitol is allowed to accumulate, but myo-inositol depletion is prevented by administration of myo-inositol, the abnormalities of NA-K-ATPase and nerve conduction are also prevented. ${ }^{10}$ Thus the NA-K-ATPase impairment in diabetic nerve is the result of myo-inositol depletion induced by sorbitol-pathway activation.

The rapidly reversible component of nerve-conduction slowing can be largely attributed to impairment of the Na-K-ATPase, which in turn is secondary to myo-inositol depletion, sorbitol accumulation, and, ultimately, hyperglycemia (Fig 4). ${ }^{12}$
Although these processes may explain the rapidly reversible slowing of nerve conduction in diabetic animals or patients, do they relate to the pathogenesis of clinically overt diabetic neuropathy? Obviously, painful paresthesias or clinically detectable sensory deficits are not easily explained simply on the basis of sodium pumps that are turned off. More likely, symptomatic diabetic neuropathy reflects the loss or severe damage to the majority of peripheral nerve fibers in patients with long-standing diabetes. ${ }^{5}$

Does the biochemical sequence clarify the development of severe structural abnormalities in peripheral nerve? Because the sodium pump plays a major role in the maintenance of the intracellular ionic environment, it is understandable how impairment of Na-K-AtPase function would lead to the swelling of cells. Indeed, the swelling of nerve fibers in the nodal and paranodal regions is the earliest structural lesion that occurs in diabetic rats. ${ }^{13}$ Persistent swelling is thought to lead to subsequent disruption of the structural elements of the nodes of Ranvier (Fig 5). ${ }^{14}$ Under normal conditions, the myelin sheath is tightly applied to the axon membrane in the paranodal region, thereby maintaining the sodium channels in high concentration there. Persistent swelling of the nodes of Ranvier is associated with loss of these axo-glial junctions, which leads to early paranodal demyelination and loss of sodium channels from the nodal region (Fig 5). This is a relatively poorly reversible structural lesion, since once these channels are lost, metabolic correction does not rapidly restore their normal distribution, and hence nodal function and structural integrity remain impaired. ${ }^{14}$

Does this sequence of events occur in human patients with diabetic neuropathy? As part of a recent multicenter trial of

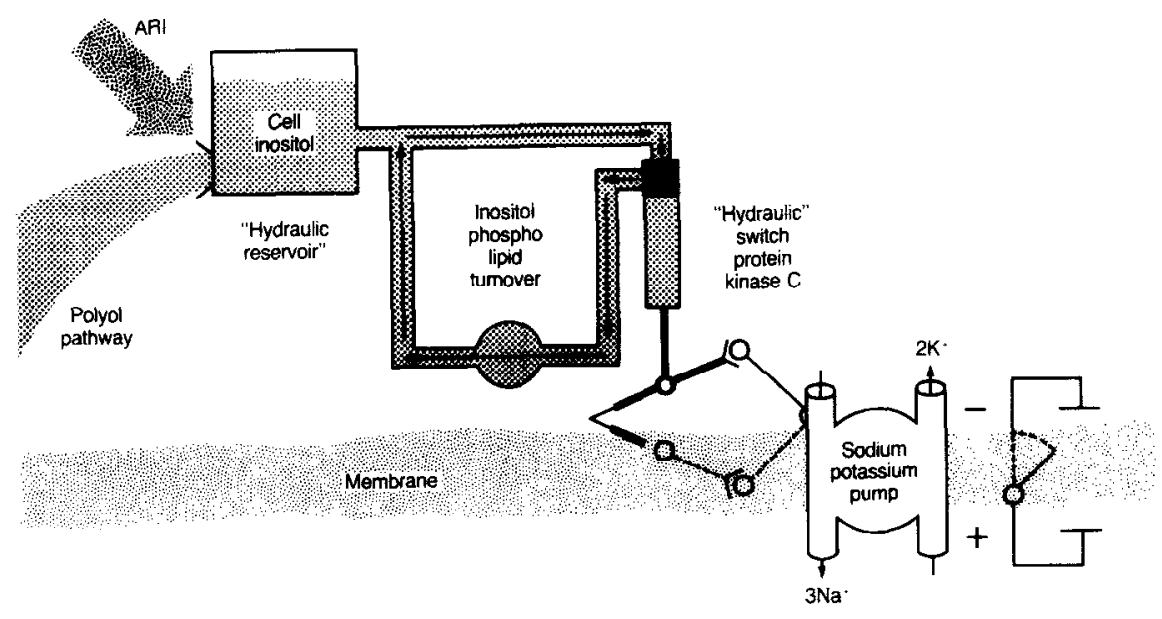

Fig 4. A Schwann cell, or axon, or both, in which hyperglycemia leads to activation of the polyol pethway. depleting some component of cell inositol. Turnover of myo-inositol through the inoaitol-phospholipid cycle is thereby diminished, reducing the release of the second messengers such as diacylglycerol. This leads to decreased activation of protein kinase C, shown here as a "hydraulic switch" that somehow turns $\mathrm{Na}-\mathrm{K}-\mathrm{ATPase}$ on and off. With Na-K-ATPase off, it fails to maintain a normal sodium gradient across the cell membrane, diminishing the current flux when voltage-dependent sodium channels are opened, leading to impaired nerve conduction. 


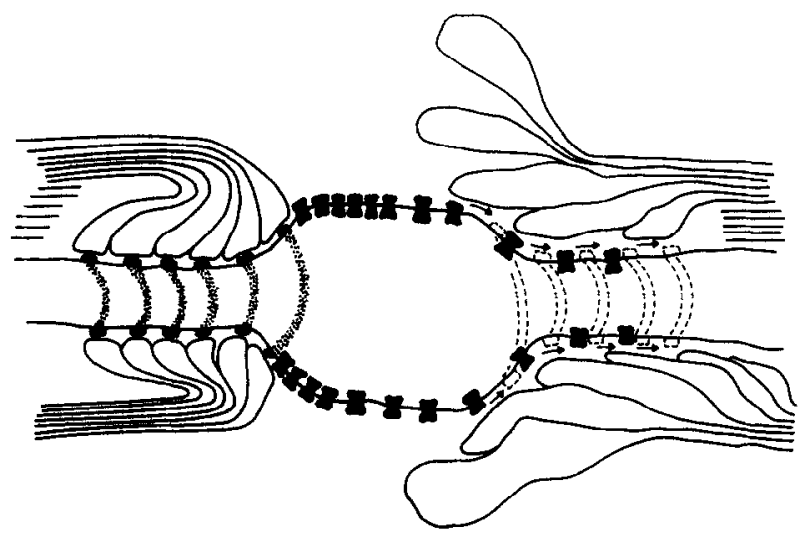

Fig 5. A node of Ranvier with normal axo-glial junctional complexes (stippled bands on left) and with disrupted axo-glial junctions (axo-glial disjunction, broken lines on right) that follow nodal swelling in insulin-deficient biobreeding rats. Voltegedependent sodium channels are shown migrating through the disrupted axo-glial junctional complexes and into the internodal region of the axolemma (arrows). Reprinted with permission. ${ }^{10}$

an aldose reductase inhibitor, nerve biopsies from subjects with diabetic neuropathy were examined for paranodal swelling and axo-glial disjunction. Both were found, although the presence of axo-glial disjunction was restricted to patients with insulin-dependent diabetes. ${ }^{15}$ Those with non-insulin-dependent diabetes exhibited additional lesions, more suggestive of an ischemic component having contributed to their neuropathy.

Histochemical studies localizing aldose reductase in nerve suggest that it is concentrated not only in the nodal region of myelinated nerve fibers, but also in the endoneurial vasculature, particularly the microvascular endothelial cell and pericyte. ${ }^{9}$ Thus, the same initiating factors (hyperglycemia, sorbitol accumulation, and myo-inositol depletion) may act at more than one tissue site within peripheral nerve, and the relative importance of these tissue sites may differ between insulin-dependent and non-insulin-dependent diabetes, perhaps because of differences in coexistent macrovascular disease.

\section{ALDOSE REDUCTASE INHIBITORS}

If a common mechanism is involved in the pathogenesis of neuropathy in insulin-dependent and non-insulin-dependent diabetes, then interruption of that mechanism might yield important therapeutic results. Preliminary data suggest that patients with diabetic retinopathy who are treated with aldose reductase inhibitors for 12 months exhibit significant improvement in their abnormal biochemistry and peripheral nerve morphology. ${ }^{16,17}$ Unexpectedly, nerve fiber repair was accompanied by a brisk, early nerve fiber regeneration. The immaturity of these fibers makes it unlikely that an immediate clinical effect would be detectable; however, extrapolation of the changes with more prolonged treatment should lead to a marked increase in the number of nerve fibers and, perhaps, reversal of clinical neurologic defects. ${ }^{17}$ Heretofore this outcome was considered highly unlikely in patients with advanced, clinically overt diabetic neuropathy.

Although the ultimate clinical effects of this treatment are still under investigation, this is probably the first example of reversal of a clinically overt complication of diabetes using a treatment directed against a specific sequela to hyperglycemia.

Thus, in peripheral nerve, hyperglycemia through activation of the polyol pathway leads to changes in myo-inositol and phosphoinositide metabolism that impairs $\mathrm{Na}-\mathrm{K}$ ATPase activity, leading to swelling, damage, and ultimately loss of nerve fibers. These same biochemical processes may also blunt the cells' ability to respond to the neurotropic factors that normally stimulate nerve regeneration in the presence of ongoing nerve damage. Correcting this inositolphosphoinositide abnormality would therefore enhance response to the neurotropic factors that usually stimulate nerve cell regeneration. These biochemical, biophysic, and neuroanatomic abnormalities provide a basis on which to build an understanding of the pathogenesis and variability of diabetic neuropathy and its slow response to treatment when in its fully developed form.

A similar pattern can be discerned in diabetic nephropathy. ${ }^{18}$ Renal disease affects non-insulin-dependent diabetics less than those who are insulin dependent, with fewer than $10 \%$ of the former experiencing renal deficiency after 20 years of disease. Indeed, in both animals with experimental diabetes and in humans with insulin-dependent diabetes, the major early functional abnormality is a hyperfunction of the kidney, with increascd renal blood flow and hyperfiltration. The early increase in glomerular filtration rate (GFR) in patients with diabetic nephropathy is blood glucose-dependent, and Goldfarb et al demonstrated that administration of an aldose reductase inhibitor or myo-inositol prevents increased GFR, ${ }^{19}$ which is thought to be a contributing factor to the subsequent development of clinical nephropathy. Similarly, aldose reductase is highly localized to the retinal pericyte, the cell that exhibits the earliest structural change in diabetic retinopathy, ${ }^{20}$ and alterations in myo-inositol metabolism and Na-K-ATPase function have also been identified in diabetic retina. ${ }^{21,22}$

\section{SUMMARY}

Thus, three complications, neuropathy, nephropathy, and retinopathy, exhibit a logical sequence that starts with hyperglycemia and proceeds towards clinically overt complications. In the case of diabetic neuropathy, it appears that specific interruption of the biochemical abnormalities is likely to lead not only to stabilization, but also to at least partial reversal of this clinically overt complication of diabetes. The possibility that therapies that interrupt this biochemical sequence may become important elements in the prevention and reversal of the three major diabetic complications holds great promise for the future.

\section{REFERENCES}

1. The DCCT Research Group: Factors in the development of diabetic neuropathy: Baseline analysis of neuropathy in the feasibil- ity phase of the Diabetes Control and Complications Trial (DCCT). Diabetes (in press) 
2. McCulloch DK, Campbell IW, Prescott RJ, et al: Effect of alcohol intake on symptomatic peripheral neuropathy in diabetic men. Diabetes Care 3:245-247, 1980

3. Steel JM, Young RJ, Lloyd GG, et al: Clinically apparent eating disorders in young diabetic women: Associations with painful neuropathy and other complications. Br Med J 294:859-862, 1987

4. Asbury AK: Focal and multifocal neuropathies of diabetes, in Dyck PJ, Thomas PK, Winegrad AI, et al (eds): Diabetic Neuropathy. Philadelphia, Saunders, 1987, pp 45-50

5. Thomas PK, Brown MJ: Diabetic polyneuropathy, in Dyck PJ, Thomas PK, Winegrad AI, et al (eds): Diabetic Neuropathy. Philadelphia, Saunders, 1987, pp 56-65

6. Dyck PJ: Pathology, in Dyck PJ, Thomas PK, Winegrad AI, et al (eds): Diabetic Neuropathy. Philadelphia, Saunders, 1987, pp 223-236

7. Troni W, Carta Q, Cantello R, et al: Peripheral nerve function and metabolic control in diabetes mellitus. Ann Neurol 16:178-183, 1984

8. Greene DA: Glycemia control, Dyck PJ, Thomas PK, Winegrad AI, et al (eds): Diabetic Neuropathy. Philadelphia, Saunders, 1987, pp 177-187

9. Chakrabarti S, Sima AAF, Nakajima T, et al: Aldose reductase in the $B B$ rat: Isolation, immunological identification and localization in the retina and peripheral nerve. Diabetologia 30:244251,1987

10. Greene DA, Lattimer SA, Sima AAF: Sorbitol, phosphoinositides, and the sodium-potassium ATPase in the pathogenesis of diabetic complications. N Engl J Med 316:599-606, 1987

11. Greene DA, Lattimer SA, Ulbrecht JS, et al: Glucoseinduced alterations in nerve metabolism: Current perspective on the pathogenesis of diabetic neuropathy and future directions for research and therapy. Diabetes Care 8:290-299, 1985

12. Brismar T, Sima AAF, Greene DA: Reversible and irreversible nodal dysfunction in diabetic neuropathy. Ann Neurol 21:504507,1987

13. Greene DA, Chakrabarti S, Lattimer SA, et al: Role of sorbitol accumulation and myo-inositol depletion in paranodal swelling of large myelinated nerve fibers in the insulin-deficient spontaneously diabetic bio-breeding rat: Reversal by insulin replacement, an aldose reductase inhibitor, and myo-inositol. J Clin Invest 79:1479-1485, 1987

14. Sima AAF, Lattimer SA, Yagihashi S, et al: "Axo-glial dysjunction," a novel structural lesion that accounts for poorly reversible slowing of nerve conduction in the spontaneously diabetic BB rat. J Clin Invest 77:474-484, 1986

15. Sima AAF, Nathaniel V, Bril V, et al: Histopathological heterogeneity of neuropathy in insulin-dependent and non-insulin dependent diabetes, and demonstration of axo-glial dysjunction in human diabetic neuropathy. J Clin Invest, (in press)

16. Greene DA, Bril V, Lattimer SA, et al: Correction of myo-inositol depletion in diabetic human sural nerve by treatment with an aldose reductase inhibitor. Diabetes 36:343A, 1987

17. Greene DA, Bril V, Sima AAF: Aldose reductase inhibitors promote sural nerve fiber regeneration and repair in patients with diabetic neuropathy. Clin Res 35:623A, 1987

18. Greene DA: Diabetic complications: Nephropathy. Drug Therapy 17:33-42, 1987

19. Goldfarb S, Kern E, Simmons D: Differential effects of supplementary dietary inositol on glomerular hyperfiltration due to early diabetes and high protein diet. Clin Res 35:623A, 1987

20. Akagi Y, Kador PF, Kuwabara T, et al: Aldose reductase localization in human retinal mural cells. Invest Ophthalmol Vis Sci 24:1516-1519, 1983

21. MacGregor LC, Rosecan LR, Laties AM, et al: Altered retinal metabolism in diabetes: I. Microanalysis of lipid, glucose, sorbitol, and myo-inositol in the choroid and in the individual layers of the rabbit retina. J Biol Chem 261:4046-4051, 1986

22. MacGregor LC, Matschinsky FM: Altered retinal metabolism in diabetes: II. Measurement of sodium-potassium ATPase and total sodium and potassium in individual retinal layers. $J$ Biol Chem $261: 4052-4058,1986$ 